\title{
Oral health status in patients with mucopolysaccharidoses
}

\author{
Elif Ballıkaya ${ }^{1}$, Pınar Serdar Eymirli ${ }^{1}$, Yılmaz Yıldız², Nihal Avcu ${ }^{3}$, Hatice Serap Sivri², \\ Meryem Uzamış-Tekçiçek ${ }^{1}$ \\ Departments of ${ }^{1}$ Pediatric Dentistry, ${ }^{3}$ Dentomaxillofacial Radiology, Hacettepe University Faculty of Dentistry, Ankara; \\ ${ }^{2}$ Division of Pediatric Metabolism, Department of Pediatrics, Hacettepe University, Ankara, Turkey. E-mail: eyildirim@ \\ hacettepe.edu.tr \\ Received: 4th July 2018, Revised: 12th July 2018, Accepted: 14th July 2018
}

SUMMARY: Ballıkaya E, Eymirli PS, Yıldız Y, Avcu N, Sivri HS, UzamışTekçiçek M. Oral health status in patients with mucopolysaccharidoses. Turk J Pediatr 2018; 60: 400-406.

The aim of this study is to assess oral clinical and radiological findings of patients with mucopolysaccharidoses (MPS) which are a group of rare inherited disorders of glycosaminoglycan catabolism. In this retrospective and descriptive study, records of all patients with MPS over 3 years of age managed at Division of Pediatric Metabolism, who were all referred to Department of Pediatric Dentistry for evaluation of their oral health status were assessed. Oral examinations were performed in 30 patients with determination of $\mathrm{DMFT} / \mathrm{S}, \mathrm{dmft} / \mathrm{s}$, dental plaque and gingival indices and panoramic radiological assessment was performed in 12 patients. Twenty-seven $(90.0 \%)$ patients had dental caries and 19/21 (90.5\%) had gingival inflammation. Radiological examination in 12 patients with panoramic image showed short mandibular ramus $(100.0 \%)$, enlarged dental follicles $(83.3 \%)$, taurodontism $(75.0 \%)$, flat mandibular notch $(66.7 \%)$. In association with the bone structure and morphology of the jaws, 11 patients $(91.7 \%)$ had trabecular variation similar to osteoporotic disease. Condylar defect was observed in all patients, which might lead to temporomandibular joint disorders. Pulp chamber with three horns, which had never been reported in MPS before, was observed in panoramic images of 2 of 7 patients with MPS-VI. Oral clinical and radiological changes are very common in this population of MPS patients. These changes may impair opening of the mouth, eating and chewing and thus affect their quality of life. Regular dental counseling, preventive applications and dental treatments must be included in multidisciplinary team approach of MPS patients to provide better oral health.

Key words: mucopolysaccharidosis, dentistry, oral health, panoramic oral radiology.

Mucopolysaccharidoses (MPSs) are a group of heterogeneous, progressive, multisystemic lysosomal storage disorders caused by deficiencies of several lysosomal enzymes required for glycosaminoglycan (GAG) breakdown. The overall prevalence of the 7 distinct types of MPS (I, II, III, IV, VI, VII, and IX) may range from 1.9 to 4.5 per 100,000 live births, with regional variations. ${ }^{1-5}$ It is expected to be higher in Turkey than that of the developed world due to the high rate of consanguineous marriages $(25 \%){ }^{6}$

Although clinical findings vary with the type of MPS, main clinical features include short stature, macrocephaly, characteristic coarse facies, umbilical and inguinal hernias, developmental delay, skeletal dysplasia with dysostosis multiplex, limited joint mobility or joint laxity, hearing loss, ocular involvement, neurodegeneration with dementia, heart disease, respiratory distress and hepatosplenomegaly. ${ }^{7-9}$ Therefore, a multidisciplinary treatment approach is essential for the diagnosis, treatment, and follow-up of patients with MPS. ${ }^{10}$ MPS patients also present with oral findings that can be observed clinically or radiologically. Oral features commonly reported in MPS include thick lips, anterior open bite, 
gingival hyperplasia, diastemas, macroglossia, high palate and condylar defects. ${ }^{11-15} \mathrm{~A}$ higher prevalence of dental caries, malocclusion, poor periodontal health, and delayed eruption of teeth, cystic lesions or enlargement of the dental follicle may also be present. ${ }^{12,13,16}$

Patients with MPS are a challenge for dentists due to complicated mental and physical disabilities. If treatment under general anesthesia becomes necessary, oral health providers need to be aware of associated risks of anesthesia due to difficulties with airway management and comorbid multisystemic involvement, especially cardiorespiratory problems. ${ }^{17}$

Dentists may encounter patients with MPS because of orofacial findings. Greater awareness of MPS will enable early referral, diagnosis and treatment, which may improve patients' quality of life and illness outcomes, since specific treatment modalities including enzyme replacement therapy and hematopoietic stem cell transplantation are available for some types of MPS. The present study aimed to investigate the oral health status of 30 MPS patients followed in a single center.

\section{Material and Methods}

This retrospective and descriptive study was performed by the approval of the Local Ethical Committee of Non-Interventional Clinical Studies in compliance with the Helsinki Declaration (Project No: GO 17/774). We assessed the records of all patients with MPS managed at a University Hospital, Faculty of Medicine, Division of Pediatric Metabolism between January 2013 and December 2015, who were all referred to Department of Pediatric Dentistry for evaluation of their oral health status. The sample consisted of all the patients whose parents allowed them to participate in the study by signing an informed consent. MPS patients over 3 years of age with complete primary dentition were evaluated; whereas those with uncooperative behavior were excluded.

Data were obtained from interview, clinical and radiological evaluations. The parents completed an oral health questionnaire performed by two investigators for each patient at the time of the dental examinations. Questions in relation to the type of MPS, age, gender and presence of other comorbid illnesses, complaints about oral health, tooth-brushing frequency were included in the questionnaire. Clinical evaluations were performed by one of two experienced pediatric dentists. Anterior open bite, dental caries, plaque index and gingival inflammation were noted by clinical examination. The total number

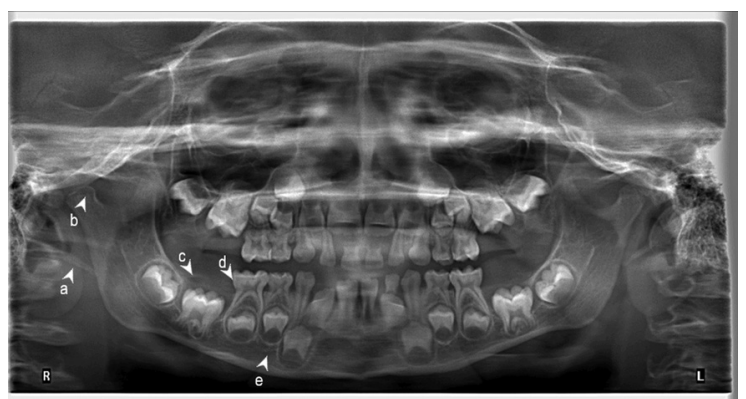

Fig. 1. Radiological findings in a patient with MPS-VI (patient no.1): malformed styloid ligament (a), (Eagle's syndrome), condylar defect (b), enlarged dental follicle (c: $46,36,26$ ), pulp chamber with three horns (d: 75,85 ), trabecular variation like osteoporotic bone (e).

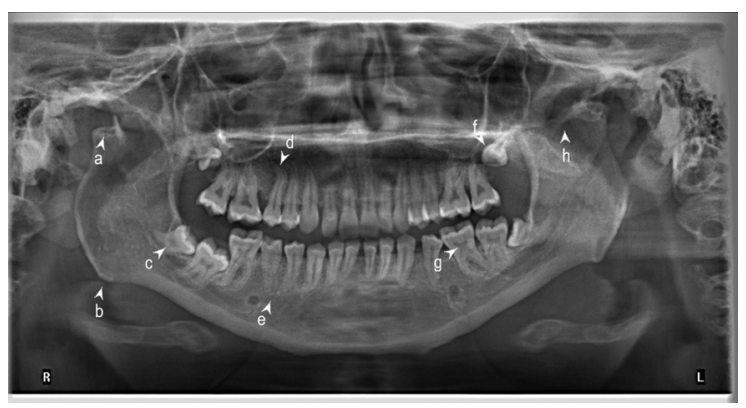

Fig. 2. Radiological findings in a patient with MPSVI (patient no. 7): condylar defect (a), prominent mandibular angulus (b), impacted teeth (c: 47, 48, 28, 18), root dilacerations (d: $15,36,46)$, trabecular variation like osteoporotic bone (e), ectopic teeth (f: 18, 28), taurodontism (g: 16, 26, 36, 46, 17, 27), flat mandibular notch (h).

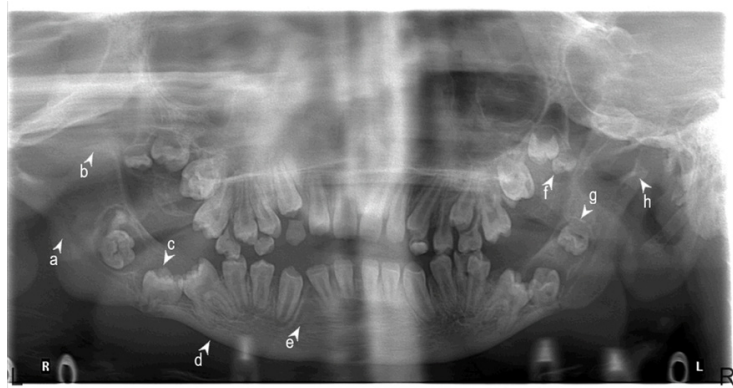

Fig. 3. Radiological findings in a patient with MPS-II (patient no.12): short mandibular ramus (a), malformed coronoid process (b), impacted teeth (c: $36,46,47,37$ ), enlarged dental follicle (c: $37,38,47,48)$, thin mandibular inferior cortex (d), trabecular variation like osteoporotic bone (e), supernumerary and ectopic teeth (f, g), condylar defect (h). 
Table I. General Characteristics of Patients with Mucopolysaccharidoses (MPS).

\begin{tabular}{lcc}
\hline Characteristics $(\mathrm{n}=30)$ & $\mathrm{n}$ & $\%$ \\
\hline Age, years & 11 & 36.7 \\
$4-6$ & 15 & 50.0 \\
$7-11$ & 4 & 13.3 \\
$14-27$ & & \\
Gender & 13 & 43.3 \\
Female & 17 & 56.7 \\
Male & & \\
MPS type & 2 & 6.7 \\
MPS-I & 3 & 10.0 \\
MPS-II & 4 & 13.3 \\
MPS-IIIA & 2 & 6.7 \\
MPS-IIIB & 6 & 20.0 \\
MPS-IVA & 12 & 40.0 \\
MPS-VI & 1 & 3.3 \\
MPS-VII & 23 & 76.7 \\
Co-morbid illnesses & 15 & 50.0 \\
Intellectual disability & 27 & 90.0 \\
Parental consanguinity & 13 & 43.3 \\
Sibling with MPS & & \\
\hline
\end{tabular}

of decayed, missing and filled teeth (DMFT/ $\mathrm{dmft}$ ) and surfaces (DMFS/dmfs) were recorded for each patient using a mirror and explorer in accordance with World Health Organization criteria and methods. Dmft/s for primary teeth, $\mathrm{DMFT} / \mathrm{S}$ codes for permanent teeth are involved in. Gingival index of Silness-Löe for gingival inflammation were used. ${ }^{18,19}$

Digital panoramic images were obtained by Veraview IC5 device (Morita Corporation, Japan) to help in the diagnosis and dental treatment planning. Eighteen patients could not cooperate for obtaining a panoramic image, therefore a total of 12 patients could be evaluated with panoramic images by a single experienced radiologist for the presence of root dilacerations, taurodontism, impacted teeth, enlarged dental follicle, delayed tooth eruption, diastema, pulpal obliteration, short mandibular ramus, irregular mandibular cortex, malformed coronoid process, malformed styloid ligament, condylar defect, a flat mandibular notch and trabecular variation. Delayed tooth eruption and diastema were also supported clinically. Statistical analysis was performed by using SPSS for Windows 21.0 (IBM Corp. Released 2012. Armonk, NY: IBM Corp.). Number, percentage, mean, standard deviation, median, 1 st and 3rd quartiles, minimum and maximum values were estimated for descriptive statistics. The normality of the distributions was analyzed by Shapiro-Wilk test.

\section{Results}

Table I shows the general characteristics of the patients. We evaluated 30 patients (17 males and 13 females) with the median age was 8 years (minimum-maximum: 4-27; 1 st quartile 6.0 years, 3rd quartile 10 years). All patients were evaluated clinically but only 12 patients could be evaluated radiologically. It was observed that a greater number of patients had MPS-VI $40 \%(n=12)$ and parental consanguinity was present in $90 \%(n=27)$ of the patients and $43.3 \%$ had a sibling with MPS. A higher number of males $(56.7 \%)$ were observed especially for MPS-II (100\%) and MPS-VI (66.7\%). Intellectual disability and other comorbid illnesses were recorded in $50 \%$ and $76.7 \%$ of patients, respectively. $43 \%$ 
Table II. Oral Health Indices of Patients with Mucopolysaccharidoses.

\begin{tabular}{cccc}
\hline Dental health indices & \multicolumn{3}{c}{ Gingival health indices } \\
\hline $\operatorname{dmft}\left(\mathrm{n}=26^{*}\right)$ & $5.50(0.0-14.00)$ & Plaque index $\left(\mathrm{n}=21^{* * *}\right)$ & $1.09(0.63-2.24)$ \\
$\operatorname{dmfs}\left(\mathrm{n}=26^{*}\right)$ & $8.00(0.0-57.00)$ & & \\
DMFT $\left(\mathrm{n}=17^{* *}\right)$ & $0.0(0.0-4.00)$ & Gingival index $\left(\mathrm{n}=21^{* * *}\right)$ & $1.38(0.03-2.43)$ \\
DMFS $\left(\mathrm{n}=17^{* *}\right)$ & $0.0(0.0-16.00)$ & & \\
\hline
\end{tabular}

The total number of decayed, missing and filled teeth (DMFT/ dmft) and surfaces (DMFS/dmfs) were recorded. Dmft/s for primary teeth, DMFT/S codes for permanent teeth are involved in.

Data is presented as median (minimum-maximum)

*dmft/dmfs values could not be evaluated in 4 patients due to permanent dentition.

**DMFT/DMFS values could not be evaluated in 9 patients due to primary dentition.

***9 patients could not be evaluated due to uncooperative behavior. PI and GI values were evaluated only in 21 cooperative patients.

$(13 / 30)$ of patients did not have a toothbrush and they stated having never brushed their teeth. Most commonly reported oral health complaints were mouth breathing (16/30) followed by tooth decay (14/30).

Clinically, 27/30 (90.0\%) patients had dental caries and $19 / 21(90.5 \%)$ had gingival inflammation. The quantity of plaque and gingival bleeding of 21 patients were checked clinically and were recorded using Silness and Löe plaque index (PI) and gingival index (GI). The median, minimum and maximum values of DMFT, DMFS, dmft, dmfs, PI, GI indices are presented in Table II.

Radiological examination in 12 patients with panoramic image showed short mandibular ramus $(100 \%)$, condylar defect $(100 \%)$, enlarged dental follicles (83.3\%), taurodontism (75\%), a flat mandibular notch (66.7\%) (Fig.1; Fig.2). Pulp chamber with three horns were observed in primary teeth of two patients. Anterior open bite (46.7\%) was present in about half of all patients. In association with the bone structure and morphology of the jaws, 11 patients $(91.7 \%)$ were observed to have trabecular variation similar to osteoporotic disease. The inferior cortex of the mandible was thinner in one patient with MPS-I, one with MPS-II and five with MPS-VI (Fig.3). Condylar defect such as abnormal morphology of condyles was observed in all patients and malformed coronoid process in 7 patients, which might lead to temporomandibular joint abnormalities.

A summary of findings from the panoramic images is presented in Table III.

\section{Discussion}

Epidemiological surveys concerning all types of MPS are still very scarce because of the low prevalence of these diseases. ${ }^{8}$ Data on MPS prevalence in Turkey is not available, but it is expected to be higher than that of the developed world, due to the high rate of consanguineous marriages (25\%). ${ }^{6}$ Parental consanguinity was present in $90 \%(n=27)$ of our patients.

The more common types of MPS in different populations have shown considerable variation. ${ }^{3}$ MPS-VI was the most common type in this study, similar to the Turkish patient population living in Germany. ${ }^{3}$ Many of our patients (76.7\%) had comorbid conditions such as cardiorespiratory, visual or hearing problems secondary to underlying MPS. Early diagnosis and multidisciplinary treatment approach are essential because many patients with MPS are at risk of multisystem impairment and significant morbidity, including dental problems. ${ }^{20}$

In the present study, majority of the patients with MPS had serious dental caries and gingival inflammation. The literature contains mainly case reports regarding oral manifestations in patients with MPS. However, there are a few studies evaluating oral health status with plaque index, gingival index, DMFT/DMFS and radiologically. 8,15 Ninety percent of MPS patients in this study had dental caries, which was higher than the study by James et al. ${ }^{12}$, who reported dental caries in $50 \%$ of their MPS patients. In the present study, the average of dmft was 5.27 and the average of DMFT was2.12, whereas James et al. ${ }^{12}$ reported the 


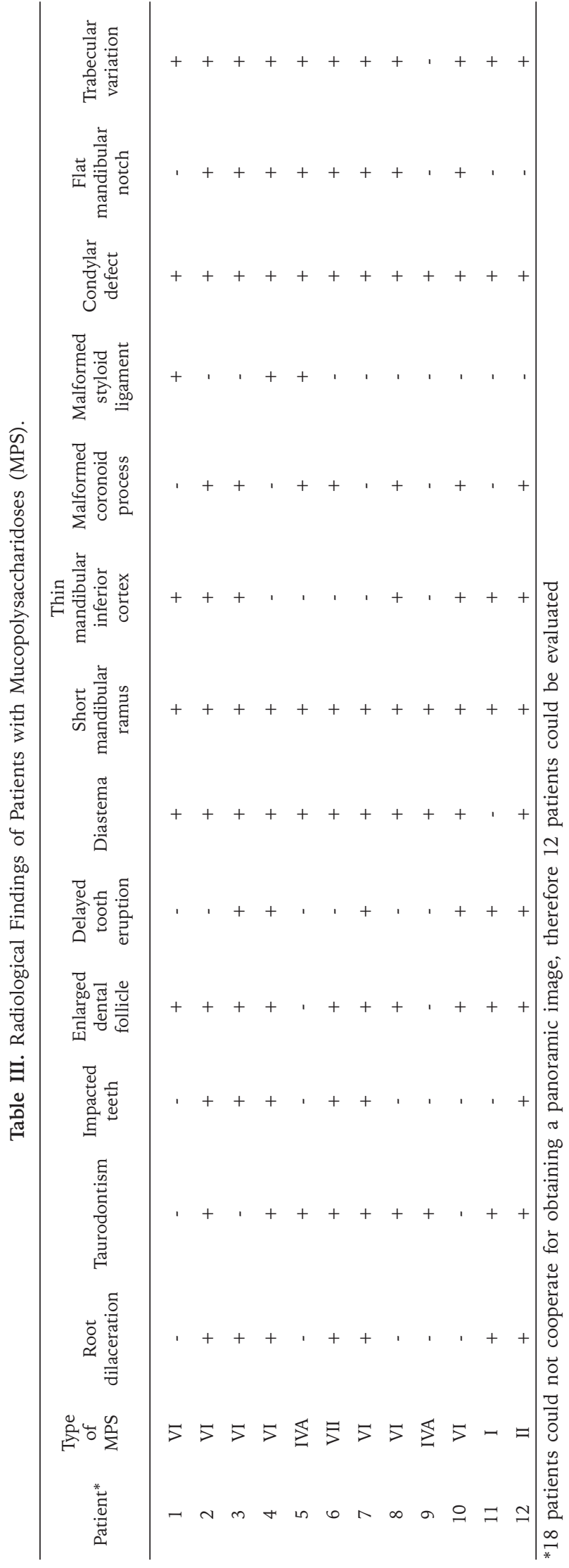

mean dmft as 3.21 and the mean DMFT as 1.67 in the MPS population. The family income and level of parental education, which was not assessed in our study as well discrepancy of oral health practices of patients, comorbid diseases, could be the reasons of the higher dental caries indices compared to the study by James et al. ${ }^{12}$

It was reported that the increase in caries in MPS patients may be associated with a decrease in salivary flow rate and more acidic $\mathrm{pH}$ than in controls. Salivary flow rate and salivary $\mathrm{pH}$ were not evaluated in our study. However, $50 \%$ of our patients have intellectual disability as well as a problem of manual dexterity and nearly half of the patients do not brush their teeth. $53.3 \%$ of the patients also complained about mouth breathing, which may have contributed to poor hygiene of the mouth and consequently, tooth decay. All these factors may explain the high prevalence of caries in our MPS patients.

The mean plaque index and gingival index $(1.30 \pm 0.48,1.22 \pm 0.65)$ showed moderate plaque accumulation and moderate gingival inflammation requiring regular oral hygiene and plaque removal. While $90.5 \%$ of 21 MPS patients showed gingivitis in the present study, James et al. ${ }^{12}$ reported $43 \%$ of MPS patients exhibited gingivitis. Elanor et al. ${ }^{21}$ reported 17 of 25 MPS patients needed periodontal treatment requiring oral hygiene, plaque or calculus removal.

Clinically, some findings such as microdontia $15,21,22$ and presence of tooth abrasion (occlusal wear) 23 , flat and wide palate $^{21}$, high-arched palate ${ }^{13,22}$ enlargement of alveolar process ${ }^{13,21,22}$, macroglossia ${ }^{13,21}$ tongue protrusion $^{24}$ thick lips ${ }^{21}$ enamel hypoplasia ${ }^{14,21,25}$ and gingival hyperplasia13,15,22-24 abnormal frenulum ${ }^{15}$ were not evaluated in the present study. However, other clinical manifestations such as anterior open-bite ${ }^{15,23}$, diastemas ${ }^{15,21,22}$, and delayed eruption ${ }^{13,15,24}$, were observed in both present study and other studies.

All MPS patients with radiological evaluation in our study (12/12) had short mandibular ramus and condylar defect. Nakamura et al. ${ }^{26}$ reported that $71 \%(29 / 41)$ of patients with MPS were observed with a condylar defect. With regard to radiological findings of other studies; impacted teeth ${ }^{8,27}$, taurodontism ${ }^{14,15,27}$, changes in coronoid process $^{8}$, delayed dental 
eruption ${ }^{8,27}$ and enlarged dental follicle $8,24,27$ were observed as reported in the literature. Other radiological manifestations described in the literature such as pulp obliteration $8,14,28$, cervical constriction ${ }^{21}$, root shortening ${ }^{17,21}$, delay in germ formation ${ }^{8}$, were not detected in the present study. However, trabecular variations like osteoporotic bone ${ }^{24,27}$, malformed styloid ligament ${ }^{21}$ and mesioangular tipping of impacted teeth and root dilacerations ${ }^{8}$, and thin mandibular inferior cortex, ${ }^{24,27}$ which were observed in our study have rarely been reported in the literature.

We realized pulp chamber with three horns in primary teeth of two patients, which had not been reported previously in MPS. We observed trabecular variation (such as enlargement of bone marrow) in $91.6 \%$ of the MPS patients and thin mandibular inferior cortex in $58.3 \%$ of the patients, which was slightly more common than that observed by Sarmento et al. ${ }^{27}$ (50\%). The accumulation of glycosaminoglycans in the bone tissues of the jaws leads to these features, which were previously reported in a case study. ${ }^{24} \mathrm{~A}$ flat mandibular notch was present in $66.6 \%$ of our patients, in line with a report by Antunes et al. ${ }^{8}$

The results of this study should be interpreted with awareness of its limitations. As this was a non-representative descriptive study that enrolled the patients who visited the metabolic clinic during a certain time period, the patients may not represent the whole MPS population. Factors such as the level of parental education and family income, which can influence the child's oral health status were not evaluated in this study. Prospective cohort studies with control groups may be required to elucidate whether mucopolysaccharidoses have a causal relationship with gingival inflammation or dental caries.

Since these patients do require special care, it is essential to educate and motivate the parents as well as the patients to provide a good oral hygiene. Complex procedures requiring sedation or general anesthesia may be high-risk in MPS patients due to various anatomic and cardiorespiratory problems which makes it even more crucial to promote regular visits to the dentist. Regular dental counseling, preventive applications and dental treatment must be included in the multidisciplinary team approach of MPS patients.

\section{REFERENCES}

1. Nelson J, Crowhurst J, Carey B, Greed L. Incidence of the mucopolysaccharidoses in Western Australia. Am J Med Genet A 2003; 123A: 310-313.

2. Lin HY, Lin SP, Chuang CK, et al. Incidence of the mucopolysaccharidoses in Taiwan, 1984-2004. Am J Med Genet A 2009; 149A: 960-964.

3. Baehner F, Schmiedeskamp C, Krummenauer F, et al. Cumulative incidence rates of the mucopolysaccharidoses in Germany. J Inherit Metab Dis 2005; 28: 1011-1017.

4. Vieira T, Schwartz I, Munoz V, et al. Mucopolysaccharidoses in Brazil: What happens from birth to biochemical diagnosis? Am J Med Genet A 2008; 146A: 1741-1747.

5. Meikle PJ, Hopwood JJ, Clague AE, Carey WF. Prevalence of lysosomal storage disorders. JAMA 1999; 281: 249-254.

6. Koc I. Prevalence and sociodemographic correlates of consanguineous marriages in Turkey. J Biosoc Sci 2008; 40: $137-148$.

7. Muenzer J, Wraith JE, Clarke LA; International Consensus Panel on Management and Treatment of Mucopolysaccharidosis I. Mucopolysaccharidosis I: Management and treatment guidelines. Pediatrics 2009; 123: $19-29$.

8. Antunes LAA, Nogueira APB, Castro GF, Ribeiro MG, de Souza IPR. Dental findings and oral health status in patients with mucopolysaccharidosis: A case series. Acta Odontol Scand 2013; 71: 157-167.

9. Ponciano S, Areias C, Leão-Teles E, Sampaio-Maia B. Hyposalivation, acidic saliva, decayed teeth and oral yeast prevalence in children with mucopolysaccharidosis. MedicalExpress 2015; 2: M150502

10. Muenzer J. The mucopolysaccharidoses: A heterogeneous group of disorders with variable pediatric presentations. J Pediatr 2004; 144 (Suppl 5): S27-S34.

11. de Almeida-Barros RQ, Oka SC, Pordeus AC, de Medeiros PF, Bento PM, Godoy GP. Oral and systemic manifestations of mucopolysaccharidosis type VI: A report of seven cases. Quintessence Int 2012; 43: e32-e38.

12. James A, Hendriksz CJ, Addison O. The oral health needs of children, adolescents and young adults affected by a mucopolysaccharide disorder. JIMD Rep 2012; 2: 51-58.

13. Alpöz AR, Coker M, Celen E, et al. The oral manifestations of Maroteaux-Lamy syndrome (mucopolysaccharidosis VI): A case report. Oral Surg Oral Med Oral Pathol Oral Radiol Endod 2006; 101: 632-637.

14. Guven G, Cehreli ZC, Altun C, et al. Mucopolysaccharidosis type I (Hurler syndrome): Oral and radiographic findings and ultrastructural/chemical features of enamel and dentin. Oral Surg Oral Med Oral Pathol Oral Radiol Endod 2008; 105: 72-78.

15. Kantaputra PN, Kayserili H, Güven Y, et al. Oral manifestations of 17 patients affected with mucopolysaccharidosis type VI. J Inherit Metab Dis 2014; 37: 263-268. 
16. Smith KS, Hallett KB, Hall RK, Wardrop RW, Firth N. Mucopolysaccharidosis: MPS VI and associated delayed tooth eruption. Int J Oral Maxillofac Surg 1995; 24: 176-180.

17. Wadenya RO, Stout AM, Gupta A, Monge J. Hurler syndrome: A case report of a 5-year follow-up of dental findings after bone marrow transplantation. Spec Care Dentist 2010; 30: 14-17.

18. World Health Organization, Oral Health Surveys Basic Methods. Geneva: WHO Press, 2013: 42-55.

19. Silness J, Löe H. Periodontal disease in pregnancy II. Correlation between oral hygiene and periodontal condition. Acta Odontol Scand 1964; 22: 121-135.

20. Lachman RS, Burton BK, Clarke LA, et al. Mucopolysaccharidosis IVA (Morquio A syndrome) and VI (Maroteaux-Lamy syndrome): Under-recognized and challenging to diagnose. Skeletal Radiol 2014; 43: 359-369.

21. McGovern E, Owens L, Nunn J, Bolas A, Meara AO, Fleming P. Oral features and dental health in Hurler Syndrome following hematopoietic stem cell transplantation. Int J Paediatr Dent 2010; 20: 322-329.

22. Downs AT, Crisp T, Ferretti G. Hunter's syndrome and oral manifestations: A review. Pediatr Dent 1995; 17: 98-100.
23. Rølling I, Clausen N, Nyvad B, Sindet-Pedersen S. Dental findings in three siblings with Morquio's syndrome. Int J Paediatr Dent 1999; 9: 219-224.

24. dos Santos Cavaleiro RM, Pinheiro MdGR, Pinheiro LR, et al. Dentomaxillofacial manifestations of mucopolysaccharidosis VI: Clinical and imaging findings from two cases, with an emphasis on the temporomandibular joint. Oral Surg Oral Med Oral Pathol Oral Radiol 2013; 116: e141-e148.

25. Gardner DG. The oral manifestations of Hurler's syndrome. Oral Surg Oral Med Oral Pathol 1971; 32 46-57.

26. Nakamura T, Miwa K, Kanda S, et al. Rosette formation of impacted molar teeth in mucopolysaccharidoses and related disorders. Dentomaxillofac Radiol 1992; 21: 45-49.

27. de Santana Sarmento DJ, de Carvalho SHG, Melo SLS et al. Mucopolysaccharidosis: Radiographic findings in a series of 16 cases. Oral Surg Oral Med Oral Pathol Oral Radiol 2015; 120: e240-e246.

28. Webman MS, Hirsch SA, Webman H, Stanley HR. Obliterated pulp cavities in the Sanfilippo syndrome (mucopolysaccharidosis III). Oral Surg Oral Med Oral Pathol 1977; 43: 734-738. 